\title{
Diffraction of Spherical Scalar and Vector Waves at Axial Points of a Circular Aperture and Disk
}

\author{
Keith Leon McDonald
}

Follow this and additional works at: https://scholarsarchive.byu.edu/facpub

Part of the Astrophysics and Astronomy Commons, and the Physics Commons

\section{Original Publication Citation}

Mcdonald, Keith L."Diffraction of Spherical Scalar and Vector Waves at Axial Points of a Circular Aperture and Disk." Journal of Applied Physics 33 (1962): 1944-1949.

\section{BYU ScholarsArchive Citation}

McDonald, Keith Leon, "Diffraction of Spherical Scalar and Vector Waves at Axial Points of a Circular Aperture and Disk" (1962). Faculty Publications. 810.

https://scholarsarchive.byu.edu/facpub/810 


\title{
Diffraction of Spherical Scalar and Vector Waves at Axial Points of a Circular Aperture and Disk
}

\author{
KeIth LeON MCDONAID \\ Depariment of Physics, Brigham Young University, Provo, Utah
}

(Received October 25, 1961)

\begin{abstract}
The following work treats Kottler's saltus problem of diffraction of electromagnetic waves emitted by a Hertzian oscillator source and the analogous Kirchhoff's scalar problem of waves emitted by a point source. The medium is a homogeneous isotropic dielectric. In the vector case a new exact solution of the basic integrals is presented, at axial points only, (a) behind a circular aperture in a "black" screen, and (b) behind its complementary "black" disk. The relative time-averaged intensity of energy flow is plotted for the disk only. It is shown that the scalar theory predicts considerably larger values than the electromagnetic theory for identical geometrical dispositions.
\end{abstract}

\section{INTRODUCTION}

$T$ HIS analysis treats the saltus problems of diffraction of electromagnetic and scalar waves at axial points of observation, (a) behind a circular aperture in a "black" screen, and (b) behind its complementary disk, for finite axial source distances. A new exact solution of the basic Kottler-Kirchhoff integrals is presented in the vector case. This is compared with the known exact solution of the analogous scalar problem. The work is of interest in applied physics since there exists no previous elementary electromagnetic solution; for the dipole field incident upon the infinitely conducting screen (Meixner, 1953), the numerical evaluation evidently has not been accomplished to any great extent. As for the scalar waves, the Airy solution of Fraunhofer diffraction is well known, whereas at finite source distances there is the work of Lommel and others, ${ }^{1,2}$ for Fresnel diffraction, including the approximate methods based upon the Fresnel half-period zones. For this analysis, we shall employ Mirimanov's exact solution of Kirchhoff's integral formulation, Eq. (4) below, for spherical scalar waves. ${ }^{3}$ Incident plane waves, both scalar and electromagnetic, being hardly applicable here since they correspond to infinite source distances, are only briefly referred to in the later discussion for reasons of comparison.

Kottler's formulation ${ }^{4}$ has been discussed by the author in a paper which will be referred to hereafter as the vector case (reference 5). The "black" screen is defined here in terms of the conditions imposed upon the complex vector disturbance q. Our exact solutions, which

\footnotetext{
1 M. E. Hufford and H. T. Davis, Phys. Rev. 33, 589-97 (1929).

2 W. Arkadiew, Physik Z. 14, 832 (1913).

${ }^{3}$ R. G. Mirimanov, C. R. Acad. Sci. U.R.S.S. 61, 617-20 (1948). The analogous exact solution of Kirchhoff's integral, at axial points only, for plane scalar waves normally incident upon a circular aperture has been given by C. L. Andrews, Phys. Rev. 71, $777-86$ (1947)

4 Friedrich Kottler, Ann. Physik 70, 405 (1923); Ann. Physik 71, 458 (1923).

${ }^{5}$ K. L. McDonald, J. Opt. Soc. Am. 43, 641-47 (1953). See also the sinusoidal formulation of J. A. Stratton and L. J. Chu, contained in Stratton's, Electromagnetic Theory (McGraw-Hill Book Company, Inc., New York, 1941). This development makes use of the vector analog of Green's theorem without recourse to scalar or vector potentials,
}

depend solely upon these initial assumptions, may now be compared with the usual approximate solutions which exploit the smallness of the wavelength $\lambda$.

\section{SCALAR CASE}

At points on the opposite sides of a "black" screen $S$, the perturbed wave function $u$ and its normal space derivative $\partial u / \partial n$ are discontinuous by an amount equal to the unperturbed wave function,

$$
u^{+}-u^{-}=\left.u\right|_{-} ^{+}=u_{0},\left.\frac{\partial u}{\partial n}\right|_{-} ^{+}=\frac{\partial u_{0}}{\partial n}
$$

In the present discussion $u_{0}=u_{0}(Q, t)=\left(1 / r_{0}\right) e^{i k\left(c t-r_{0}\right)}$, where $\lambda=2 \pi / k$, and $r_{0}$ is the radial distance measured from the light source $L$ to any point $Q$, as in the notation of Fig. 1. Applying Maggi's transformation,

$$
\begin{array}{r}
\iint\left\{\frac{e^{-i k r}}{r} \frac{\partial}{\partial n}\left(\frac{e^{i k\left(c t-r_{0}\right)}}{r_{0}}\right)-\frac{e^{i k\left(c t-r_{0}\right)}}{r_{0}} \frac{\partial}{\partial n}\left(\frac{e^{-i k r}}{r}\right)\right\} d S \\
=\oint_{C} \frac{\mathbf{r}_{0} \times \mathbf{r} e^{i k\left(c t-r_{0}-r\right)}}{\left(\mathbf{r}_{0} \cdot \mathbf{r}+r_{0} r\right) r_{0} r} \cdot d \mathbf{\Gamma}=-\Re(L, P)
\end{array}
$$

to the Kottler-Kirchhoff formula (cf., reference 6) the following disturbance results in accordance with (1),

$$
u(P, t)=\epsilon u_{0}(P, t)-(1 / 4 \pi) \Re(L, P) .
$$

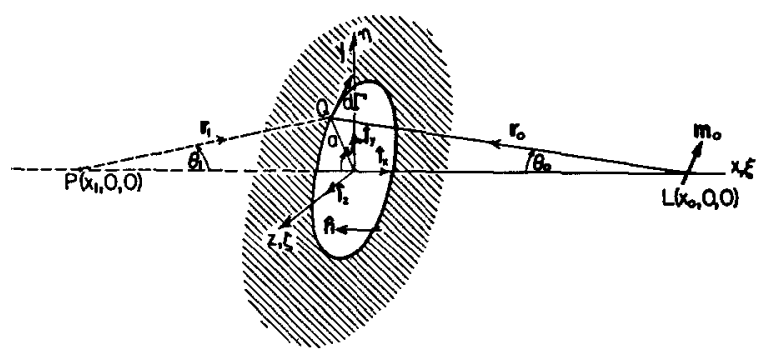

FIG. 1. Diffraction by a circular aperture in a "black" screen.

${ }^{\circ}$ B. B. Baker and E. T. Copson, The Mathematical Theory of Huygen's Principle (Oxford University Press, Oxford, England,
1950 ), 2nd ed. 
The circuital direction of $C$ is related to the positive side of the screen surface by the usual right-hand rule, and the vector $\Gamma$ connects the origin of coordinates to a peripheral point on curve $C$ bounding $S . \epsilon=0$ if the segment $L P$ intercepts the screen surface $S$; otherwise, $\epsilon=1$.

This expression is next applied to the computation of the relative time-averaged intensity of energy flow along the shadow axis of a thin circular "black" disk. Using a screen complementary to that shown, let the plane of the disk lie in the $y z$ plane, the origin of coordinates being coincident with the center of the disk, and place the source point $L$ and point of observation $P$ on the $x$ axis. The vectors $\hat{n}$ and $d \Gamma$ are therefore directed as shown. Eq. (3) gives

$$
\begin{aligned}
u(P, t)=\frac{e^{i k\left(c t-r_{0}-r_{1}\right)} \sin \left(\theta_{0}+\theta_{1}\right)}{4 \pi r_{0} r_{1}\left[1-\cos \left(\theta_{0}+\theta_{1}\right)\right]} & \int_{0}^{2 \pi} a d v \\
& =\frac{a e^{i k\left(c t-r_{0}-r_{1}\right)}}{2 r_{0} r_{1} \tan \frac{1}{2}\left(\theta_{0}+\theta_{1}\right)}
\end{aligned}
$$

$\theta, \theta_{1}$ being the angles enclosed by $r_{0}, r_{1}$, and the $z$ axis, and " $a$ " is the disk radius. In the previous value of the unperturbed disturbance at $P$, substitute $L P=r_{0} \cos \theta_{0}$ $+r_{1} \cos \theta_{1}=\left(x_{0}-x_{1}\right)$. Then the relative intensity at any point $P\left(x_{1}, 0,0\right)$ on the shadow side of the screen axis is

$$
\begin{aligned}
I / I_{0}=\frac{u u^{*}}{u_{0} u_{0}^{*}}=\frac{a^{2}\left(r_{0} \cos \theta_{0}+r_{1} \cos \theta_{1}\right)^{2}}{4 r_{0}^{2} r_{1}^{2} \tan ^{2} \frac{1}{2}\left(\theta_{0}+\theta_{1}\right)} & \\
= & \frac{1}{4}\left[1+\cos \left(\theta_{0}+\theta_{1}\right)\right]^{2} .
\end{aligned}
$$

This exact evaluation of (3) predicts a relative intensity which varies monotonically from the value $\frac{1}{4}\left(1-\sin \theta_{0}\right)^{2}$ directly behind the disk to $\frac{1}{4}\left(1+\cos \theta_{0}\right)^{2}$ at $r_{1} \doteq \infty$. Hence, the relative axial illumination is a maximum for a collimated incident beam and is everywhere independent of the wavelength $\lambda$. Figure 2 illustrates this case for both a collimated incident beam, $x_{0}=\infty$, and a finite source distance set equal to the radius of the disk; $x_{0}=a$.

If the screen is replaced by a circular aperture [for axial points $\epsilon=1 \mathrm{in} \mathrm{Eq.} \mathrm{(3)],} \mathrm{there} \mathrm{results} \mathrm{the} \mathrm{exact}$ relation

$$
\begin{aligned}
u(P, t)= & (1 / L P) e^{i k(c t-L P)} \\
& -(1 / 2 L P)\left[1+\cos \left(\theta_{0}+\theta_{1}\right)\right] e^{i k\left(c t-r_{0}-r_{0}\right)} .
\end{aligned}
$$

The relative intensity is accordingly

$$
\begin{aligned}
I / I_{0}=1+\frac{1}{4}\left[1+\cos \left(\theta_{0}+\theta_{1}\right)\right]^{2} & \\
- & {\left[1+\cos \left(\theta_{0}+\theta_{1}\right)\right] \cos k\left(r_{0}-x_{0}+r_{1}+x_{1}\right) . }
\end{aligned}
$$

Because of the last term it is evident that the relative ntensity oscillates between the lower and upper bounds

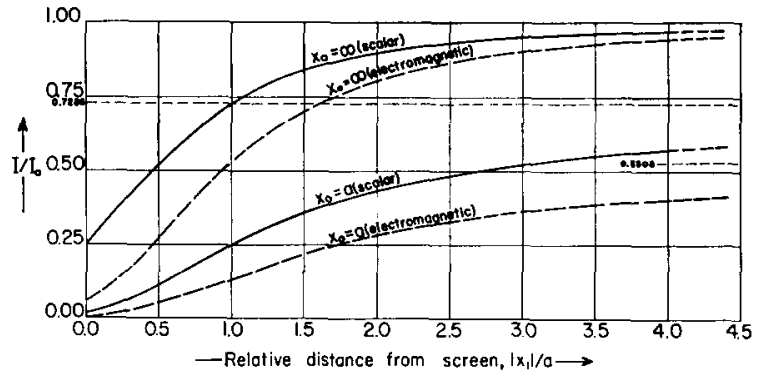

FIG. 2. Theoretical relative intensity distribution at axial points behind a "black" circular disk of radius $a$. The scalar cases pertain to all wavelengths whereas the vector cases shown are delimited to wavelengths small compared to other length factors.

of 0 and 4 as the point of observation moves along the $x$ axis. Moreover, the source distance and aperture radius affect its linear distribution. If the source is sufficiently removed so that $\theta_{0} \doteq 0$, the relative intensity varies between the limits $5 / 4 \pm|\cos (2 \pi a / \lambda)|$ at the aperture center, and for large increasing values of the radius $a$, is not equal to unity as one would require of a rigorous formalism. This deficiency is inherent in the Kottler-Kirchhoff scheme and does not arise from approximations to the basic integrals. Since the amplitude factor of the last term is a nondecreasing function of $r_{1}$, and the phase difference $2 \pi\left(r_{1}-\left|x_{1}\right|\right) / \lambda$ is a nonincreasing function, it is evident that its contribution becomes larger but that the oscillations occur less frequently as the point of observation moves away from the screen. Finally, we note that when the wavelength is increased the oscillations of the relative intensity are everywhere less frequent. These results are in qualitative agreement with the less formal predictions derived from the application of the Fresnel half-period zones. ${ }^{7,8}$

Equation (4), due to Mirimanov (reference 3), is incidental to his general argument so that the above inferences, together with the remaining equations of this section, have never been discussed. Current literature incorporates the assumption that the wavelength $\lambda$ is small compared to other length factors. Such solutions, however, are more cumbersome than the above exact expressions.

\section{VECTOR CASE}

The previous problem, treated next from the standpoint of electromagnetic theory, employs a point source consisting of a simple harmonic Hertzian oscillator placed on the aperture axis. The two important orientations of oscillator are directed along the aperture axis and perpendicular thereto. The appropriate field equations, Eqs. (7) of the vector case, are again written in

${ }^{7}$ C. F. Meyer, The Diffraction of Light, X-Rays, and Material Particles (The University of Chicago Press, Chicago, Illinois, 1934).

${ }^{8}$ F. A. Jenkins and H. E. White, Fundamentals of Optics (McGraw-Hill Book Company, Inc., New York, 1957), 3rd ed., p. 353. 
terms of Gaussian units,

$$
\begin{aligned}
& 4 \pi \mathbf{E}(P)=-\frac{c}{j \omega K} \oint_{\Gamma}\left(\nabla_{Q} \Phi_{1}\right) \mathbf{H}_{0} \cdot d \boldsymbol{\Gamma}-\oint_{\Gamma} \Phi_{1} \mathbf{E}_{0} \times d \mathbf{\Gamma} \\
& +D_{L} \frac{\mathbf{m}_{0}}{K}\left\{4 \pi \epsilon \Phi_{1}(L, P)-\Re(L, P)\right\}, \\
& 4 \pi \mathbf{H}(P)=\frac{c}{j \omega \mu} \oint_{\Gamma}\left(\nabla_{Q} \Phi_{1}\right) \mathbf{E}_{0} \cdot d \Gamma-\oint_{\Gamma} \Phi_{1} \mathbf{H}_{0} \times d \Gamma \\
& -\frac{j \omega K}{c} \nabla_{L}\left\{4 \pi \epsilon \Phi_{1}(L, P)-\Re(L, P)\right\} \times \frac{\mathbf{m}_{0}}{K}
\end{aligned}
$$

where $\mathfrak{R}$ is given by (2) or (6) of the vector case. For convenience, the following quantities are also reproduced:

$$
\begin{aligned}
\mathbf{E}_{0}(P) & =D_{P} \stackrel{\mathbf{m}_{0}}{K} \Phi_{0}(L, P) \\
\mathbf{H}_{0}(P) & =\frac{j \omega K}{c} \nabla_{P} \Phi_{0}(L, P) \times \frac{\mathbf{m}_{0}}{K} \\
D_{P} & =\Lambda^{2} \omega^{2}+\nabla_{P} \nabla_{P} . \\
\Phi_{1}(Q, P) & =\frac{1}{r_{1}} e^{-j \omega \Lambda r_{1}} \\
\Phi_{0}(L, Q) & =\frac{1}{r_{0}} e^{-j \omega \Delta r_{0}} .
\end{aligned}
$$

Figure 1 has been drawn to conform with this notation. Observe the change in notation; vector quantities are here denoted by boldface type; the functions $\phi_{0}$ and $\phi_{1}$, except whenever they occur there as polar coordinates in a circular function, are here denoted by $\Phi_{0}$ and $\Phi_{1}$. The wave slowness is again $\Lambda=\left(K \mu / c^{2}\right)^{\frac{1}{2}}, c$ being the velocity of light in free space. $\mu$ and $K$ are the permeability and specific inductive capacity of the medium, and $\mathbf{m}_{0}$ denotes the constant electric moment of the oscillator. For fields of finite past history, or whenever the source is subjected to amplitude variations (modulation), it is necessary to resort to the earlier equations in the vector case, Eqs. (2) or (3).

For axial positions both $r_{0}$ and $r_{1}$ are constants in the integration process around the periphery of the aperture. It follows that $\Phi_{0}$ and $\Phi_{1}$, as well as their total derivatives, are likewise constants. Thus they may be factored out of the integrands provided that no indicated differentiations appear in front of the integral sign; otherwise, it is necessary to displace the source and point of observation to arbitrary positions, perform the indicated operations and then evaluate the results at the original positions. This is the present procedure. It is worthwhile to include one intermediate step before writing down the solutions because the computations are quite lengthy. Although this step may be employed in later computations for extra-axial positions of $L$ and $P$, for example, by the method of stationary phase, it is likely that a direct operation on (7) would prove shorter. Recalling that

$$
\frac{\partial r_{0}}{\partial x_{Q}}=\frac{x_{Q}-x_{L}}{r_{0}}=-\frac{\partial r_{0}}{\partial x_{L}}, \text { etc. }
$$

and denoting the total derivatives of $\Phi_{0}$ and $\Phi_{1}$ by $\Phi_{0}{ }^{\prime}$ $=d \Phi_{0} / d r_{0}, \Phi_{1}^{\prime}=d \Phi_{1} / d r_{1}$, it follows that

$$
\begin{aligned}
& \nabla_{Q} \Phi_{0}=\left(1 / r_{0}\right) \Phi_{0}{ }^{\prime} \mathbf{r}_{0}=-\nabla_{L} \Phi_{0}, \\
& \nabla_{Q} \Phi_{1}=\left(1 / r_{1}\right) \Phi_{1}{ }^{\prime} \mathbf{r}_{1}=-\nabla_{P} \Phi_{1} .
\end{aligned}
$$

Using this notation and substituting the values of $\mathbf{E}_{0}, \mathbf{H}_{0}$ of (8) into (7), there obtains for the electric intensity (denoting unit vectors by caret symbol),

$$
\begin{aligned}
4 \pi \mathrm{E}(P)= & -\frac{\mathbf{m}_{0}}{K} \cdot \oint_{\Gamma} \Phi_{0}{ }^{\prime} \Phi_{0}{ }^{\prime} d \mathbf{\Gamma} \times \hat{\mathbf{r}}_{0} \hat{\mathbf{r}}_{1}-\frac{\mathbf{m}_{0}}{K} \times \oint_{\Gamma} \Phi_{1}\left[\Lambda^{2} \omega^{2} \Phi_{0}+\Phi_{0}{ }^{\prime} / r_{0}\right] d \mathbf{\Gamma} \\
-\frac{\mathbf{m}_{0}}{K} \cdot \oint_{\Gamma} r_{0} \Phi_{1} \frac{d}{d r_{0}}\left(\Phi_{0}{ }^{\prime} / r_{0}\right) \hat{\mathbf{r}}_{0} \hat{\mathbf{r}}_{0} \times d \Gamma+\frac{\mathbf{m}_{0}}{K} 4 \pi \epsilon\left[\Lambda^{2} \omega^{2} \Phi_{1}+\Phi_{1}{ }^{\prime} / r_{1}\right]_{P L}+\frac{\mathbf{m}_{0}}{K} \cdot \hat{\mathbf{r}}_{P L} \hat{\mathbf{r}}_{P L} 4 \pi \epsilon\left[r_{1} \frac{d}{d r_{1}}\left(\Phi_{1}{ }^{\prime} / r_{1}\right)\right]_{P L} & -\frac{\mathbf{m}_{0}}{K} \Lambda^{2} \omega^{2} \Re(L, P)-\frac{\mathbf{m}_{0}}{K} \cdot \nabla_{L} \nabla_{L} \Re(L, P) .
\end{aligned}
$$

The indicated differentiation in the last term is not written out since the operation is straightforward and rather lengthy. The continuity of all integrands with respect to points $L$ and $Q$ is a sufficient condition for the interchange of the operations of differentiation and integration. Introducing the idemfactor $\mathbf{I}$, the differentiated integrand contains eight symmetric dyadic terms. Forming the scalar product with $\mathbf{m}_{\mathbf{0}} / K$ as a prefactor or postfactor is therefore 
immaterial. The expression for the magnetic intensity is considerably simpler,

$$
\begin{aligned}
& 4 \pi \mathbf{H}(P)=\frac{c}{j \omega \mu} \frac{\mathbf{m}_{0}}{K} \cdot\left\{\Lambda^{2} \omega^{2} \oint_{\Gamma} \Phi_{0} \Phi_{1}{ }^{\prime} d \boldsymbol{\Gamma} \hat{\mathbf{r}}_{1}+\oint_{\Gamma} \frac{1}{r_{0}} \Phi_{0}{ }^{\prime} \Phi_{1}{ }^{\prime} d \boldsymbol{\Gamma} \hat{\mathbf{r}}_{1}+\oint_{\mathrm{r}} r_{0} \frac{d}{d r_{0}}\left(\Phi_{0}{ }^{\prime} / r_{0}\right) \hat{\mathbf{r}}_{0} \hat{\mathbf{r}}_{0} \cdot d \boldsymbol{\Gamma} \hat{\mathbf{r}}_{1}\right\} \\
& +\frac{j \omega K}{c}\left(\frac{\mathbf{m}_{0}}{K} \times\left\{\oint_{\Gamma} \Phi_{0}{ }^{\prime} \Phi_{1} \hat{\mathbf{r}}_{0}\right) \times d \mathbf{\Gamma}+\hat{\mathbf{r}}_{P L} 4 \pi \epsilon \Phi_{1}^{\prime}(L, P)+\oint_{\Gamma} \Phi_{0}{ }^{\prime} \Phi_{1} \hat{\mathbf{r}}_{0} \frac{\left(\mathbf{r}_{0} \times \mathbf{r}_{1}\right) \cdot d \boldsymbol{\Gamma}}{\left(\mathbf{r}_{0} \cdot \mathbf{r}_{1}+r_{0} \boldsymbol{r}_{1}\right)}\right. \\
& \left.+\left(\mathbf{I} \times \oint_{\Gamma} \mathbf{r}_{1}\right) \frac{\Phi_{0} \Phi_{1} \cdot d \mathbf{\Gamma}}{\left(\mathbf{r}_{0} \cdot \mathbf{r}_{1}+r_{0} r_{1}\right)}-\oint_{\Gamma} \Phi_{0} \Phi_{1} \frac{\left(\mathbf{r}_{0} r_{1}+r_{0} \mathbf{r}_{1}\right)\left(\mathbf{r}_{0} \times \mathbf{r}_{1}\right) \cdot d \mathbf{\Gamma}}{r_{0}\left[\mathbf{r}_{0} \cdot \mathbf{r}_{1}+r_{0} r_{1}\right]^{2}}\right\} .
\end{aligned}
$$

Equations (9) and (10), being applicable to apertures of quite arbitrary shape, are next specialized according to the geometrical disposition of Fig. 1. The following substitutions are necessary: $\boldsymbol{\Gamma}=\hat{\imath}_{y} \eta+\hat{\imath}_{z} \zeta, \eta=a \cos \nu, \zeta=a \sin \nu$, $\mathbf{r}_{0}=\hat{\imath}_{x}\left(-x_{0}\right)+\hat{\imath}_{y} \eta+\hat{\imath}_{z} \zeta, \mathbf{r}_{1}=\hat{\imath}_{x}\left(-x_{1}\right)+\hat{\imath}_{y} \eta+\hat{i}_{2} \zeta, \mathbf{r}_{0} \cdot d \mathbf{\Gamma}=0, \mathbf{r}_{1} \cdot d \mathbf{\Gamma}=0, \mathscr{\Phi} d \mathbf{\Gamma}=0$. Considering the simplification of (9), one is led to the following definite integrals

$$
\begin{aligned}
& \oint_{\Gamma} d \mathbf{\Gamma} \times \mathbf{r}_{0} \mathbf{r}_{1}=\pi a^{2}\left[-2 x_{1} \hat{\imath}_{z} \hat{\imath}_{x}+x_{0} \hat{\imath}_{y} \hat{\imath}_{y}+x_{0} \hat{\imath}_{z} \hat{\imath}_{z}\right] \\
& \oint_{\Gamma} \mathbf{r}_{0} \mathbf{r}_{0} \times d \mathbf{\Gamma}=\pi x_{0} a^{2}\left[2 \hat{\imath}_{x} \hat{\imath}_{x}-\hat{\imath}_{y} \hat{\imath}_{y}-\hat{\imath}_{z} \hat{\imath}_{z}\right] \\
& \oint_{\Gamma}\left[\mathbf{I} \times \mathbf{r}_{1} \cdot d \mathbf{\Gamma} \mathbf{r}_{0}+\mathbf{r}_{0} \mathbf{I} \times \mathbf{r}_{1} \cdot d \boldsymbol{\Gamma}\right]=2 \pi a^{2}\left[2 x_{0} \hat{\imath}_{x} \hat{\imath}_{x}-x_{1} \hat{\imath}_{y} \hat{\imath}_{y}-x_{1} \hat{\imath}_{z} \hat{\imath}_{z}\right] \\
& \int_{0}^{2 \pi} \mathbf{r}_{0} \mathbf{r}_{1} d \nu=\int_{0}^{2 \pi} \mathbf{r}_{1} \mathbf{r}_{0} d \nu=\pi a^{2}\left[2\left(x_{0} x_{1} / a^{2}\right) \hat{\imath}_{z} \hat{\imath}_{x}+\hat{\imath}_{y} \hat{\imath}_{y}+\hat{\imath}_{z} \hat{\imath}_{z}\right] .
\end{aligned}
$$

The remaining terms are derived from these as special cases. Performing the necessary simplifications, the total $\mathbf{E}$ vector is next written for the two principal orientations of $\mathbf{m}_{0}$.

$$
\begin{aligned}
& \pi \text {-Case }\left(\mathbf{m}_{0}=\hat{\imath}_{x} m_{0}\right): \\
& 4 \pi \mathbf{E}(P)=4 \pi \mathrm{E}_{0}(P) \epsilon+\hat{\imath}_{x} \frac{2 \pi m_{0} a}{K}\left\{\frac{a x_{1} \Phi_{0}{ }^{\prime} \Phi_{1}{ }^{\prime}}{r_{0} r_{1}}-\frac{a x_{0}}{r_{0}} \Phi_{1} \frac{d}{d r_{0}}\left(\Phi_{0}{ }^{\prime} / r_{0}\right)-\left[\Lambda^{2} \omega^{2} \Phi_{0}+\left(\Phi_{0}{ }^{\prime} / r_{0}\right)+\frac{x_{0}{ }^{2}}{r_{0}} \frac{d}{d r_{0}}\left(\Phi_{0}{ }^{\prime} / r_{0}\right)\right] \Phi_{1} \operatorname{ctn}\left(\frac{\theta_{0}+\theta_{1}}{2}\right)\right. \\
& +2 x_{0}\left(x_{1} r_{0}+x_{0} r_{1}\right) \frac{\Phi_{0}{ }^{\prime} \Phi_{1} \sin \left(\theta_{0}+\theta_{1}\right)}{r_{0}{ }^{2} r_{1}\left[1-\cos \left(\theta_{0}+\theta_{1}\right)\right]^{2}}-\frac{2 x_{0} a \Phi_{0}{ }^{\prime} \Phi_{1}}{r_{0}{ }^{2} r_{1}\left[1-\cos \left(\theta_{0}+\theta_{1}\right)\right]}+\left(r_{0}{ }^{2}-x_{0}{ }^{2}\right) \Phi_{0} \Phi_{1} \frac{\sin \left(\theta_{0}+\theta_{1}\right)}{r_{0}{ }^{4}\left[1-\cos \left(\theta_{0}+\theta_{1}\right)\right]^{2}} \\
& \left.+\frac{2 a\left(r_{1} x_{0}+r_{0} x_{1}\right) \Phi_{0} \Phi_{1}}{r_{0}^{3} r_{1}^{2}\left[1-\cos \left(\theta_{0}+\theta_{1}\right)\right]^{2}}-2\left(x_{0} r_{1}+x_{1} r_{0}\right)^{2} \Phi_{0} \Phi_{1} \frac{\sin \left(\theta_{0}+\theta_{1}\right)}{r_{0}^{4} r_{1}^{2}\left[1-\cos \left(\theta_{0}+\theta_{1}\right)\right]^{3}}\right\} .
\end{aligned}
$$

$\epsilon$ has been retained in place of unity to delineate its coefficient terms

$$
\mathbf{E}_{0}(P)=\hat{\imath}_{t_{x}} \frac{m_{0}}{K}\left[\Lambda^{2} \omega^{2} \Phi_{0}(L, P)+\Phi_{0}{ }^{\prime \prime}(L, P)\right]
$$

$\epsilon=1$, since the aperture axis lies in the geometrically illuminated region. This is the exact expression for $\mathbf{E}(P)$ when $\mathbf{m}_{0}=\hat{\imath}_{x} m_{0}$. Observe that $\mathbf{E}(P)$ is directed along the aperture axis. Consider next the more important

$$
\begin{aligned}
& \sigma \text {-Case }\left(\mathbf{m}_{0}=\hat{\imath}_{y} m_{0}\right): \\
& 4 \pi \mathbf{E}(P)=4 \pi \mathbf{E}_{0}(P) \epsilon+\hat{\imath}_{y} \frac{\pi a m_{0}}{K}\left\{-\frac{a x_{0}}{r_{0} r_{1}} \Phi^{\prime} \Phi_{1}{ }^{\prime}+\frac{a x_{0}}{r_{0}} \Phi_{1} \frac{d}{d r_{0}}\left(\Phi_{0}{ }^{\prime} / r_{0}\right)-\left[2 \Lambda^{2} \omega^{2} \Phi_{0}+\frac{a^{2}}{r_{0}} \frac{d}{d r_{0}}\left(\Phi_{0}{ }^{\prime} / r_{0}\right)+2 \Phi_{0}{ }^{\prime} / r_{0}\right] \Phi_{1} \operatorname{ctn}\left(\frac{\theta_{0}+\theta_{1}}{2}\right)\right. \\
& +\frac{2 a x_{1} \Phi_{0}{ }^{\prime} \Phi_{1}}{r_{0}{ }^{2} r_{1}\left[1-\cos \left(\theta_{0}+\theta_{1}\right)\right]}-\frac{2 a x_{1}\left(r_{0}+r_{1}\right) \Phi_{0} \Phi_{1}}{r_{0}^{3} r_{1}^{2}\left[1-\cos \left(\theta_{0}+\theta_{1}\right)\right]^{2}}+\left[2 a^{2}\left(r_{0}+r_{1}\right) r_{0} \Phi_{0}{ }^{\prime}+r_{1}\left(2 r_{0}{ }^{2}-a^{2}\right) \Phi_{0}\right]-\frac{\Phi_{1} \sin \left(\theta_{0}+\theta_{1}\right)}{r_{0}{ }^{4} r_{1}\left[1-\cos \left(\theta_{0}+\theta_{1}\right)\right]^{2}} \\
& \left.-2 a^{2}\left(r_{0}+r_{1}\right)^{2} \frac{\Phi_{0} \Phi_{1} \sin \left(\theta_{0}+\theta_{1}\right)}{r_{0}^{4} r_{1}^{2}\left[1-\cos \left(\theta_{0}+\theta_{1}\right)\right]^{3}}\right\} \text {. }
\end{aligned}
$$


Again $\epsilon=1$. Its coefficient is the unperturbed intensity

$$
4 \pi \mathrm{E}_{0}(P)=4 \pi \frac{m_{0}}{K} \hat{i}_{y}\left[\Lambda^{2} \omega^{2} \Phi_{1}(L, P)+\Phi_{1}^{\prime}(L, P) / r_{L P}\right] .
$$

Observe that $\mathbf{E}(P)$ is everywhere directed along the $y$ axis. The corresponding expressions for $\mathbf{H}(P)$ are considerably simpler. Performing the indicated operations in (10), one finds that the $\pi$-Case leads to $\mathbf{H}\left(x_{1}\right) \equiv 0, x_{1}<0$; the aperture axis is a fixed line of vector singular points of the time dependent $\mathbf{H}$ field. By symmetry the magnetic lines of force are circles about this axis. Hence, for axial points, the $\pi$-Case is trivial and will not be further discussed. Finally, in the $\sigma$-Case $\left(\mathbf{m}_{0}=\hat{\imath}_{y} m_{0}\right)$ :

$$
\begin{aligned}
4 \pi \mathbf{H}(P)=4 \pi \mathbf{H}_{0}(P) \epsilon+\hat{\imath}_{2} \frac{m_{0} \pi}{K}\left\{\frac{c}{j \omega \mu}\left[\Lambda^{2} \omega^{2} \Phi_{0}+\Phi_{0} / r_{0}\right] \frac{a^{2} \Phi_{1}{ }^{\prime}}{r_{1}}+\frac{j \omega K}{c}\left[\left(a^{2} / r_{0}\right) \Phi_{0} \Phi_{1}+2 a\left(x_{0} / r_{0}\right) \Phi_{0}{ }^{\prime} \Phi_{1} \operatorname{ctn}\left(\frac{\theta_{0}+\theta_{1}}{2}\right)\right.\right. & \\
& \left.\left.+\frac{2 a^{2} \Phi_{0} \Phi_{1}}{r_{0} r_{1}\left[1-\cos \left(\theta_{0}+\theta_{1}\right)\right]}-\frac{2 a\left(r_{0} x_{1}+r_{1} x_{0}\right) \Phi_{0} \Phi_{1}}{r_{0}{ }^{2} r_{1}\left[1-\cos \left(\theta_{0}+\theta_{1}\right)\right]} \operatorname{ctn}\left(\frac{\theta_{0}+\theta_{1}}{2}\right)\right]\right\} \\
4 \pi \mathbf{H}_{0}(P)=-\frac{j \omega K}{c} \frac{4 \pi m_{0}}{K} \Phi_{0}{ }^{\prime}(L, P) \hat{\imath}_{z} ; \quad \epsilon=1 . &
\end{aligned}
$$

Observe that $\mathbf{H}(P)$ is everywhere parallel to the $z$ axis and perpendicular to $\mathbf{E}(P)$ so that the Poynting vector is along the aperture axis. This solves the problem of diffraction by an aperture.

The solution pertaining to the complementary screen, the "black" circular disk, is now readily demonstrated by use of Eq. (3) of the vector case, which may be referred to as the aperture formula. In this paper the complex vector disturbance $\bar{q}(\equiv \mathbf{q}$ in the present notation) is obtained by carrying out the integration process over the surface $S$ of the aperture and its boundary, in accord with the right-hand rule. From this expression one may derive an alternate formula which is suited to problems in diffraction by an obstacle. By a process of continuous deformation the unperturbed field $\mathbf{q}_{0}$ is obtained from the above expression by replacing the screened surface with an aperture. The resulting contour integrals cancel and there remains only the surface integral $\mathbf{K}=4 \pi \mathbf{q}_{0}$ taken over the closed surface surrounding the source distribution. The result is the wellknown Larmor-Tedone equation,

$$
\begin{aligned}
4 \pi \mathbf{q}_{0}(P, t)=\iint\left\{\left[\mathbf{q}_{0}\right] \frac{\partial}{\partial n} \frac{1}{r_{1}}\right. & -\frac{1}{r_{1}}\left[\frac{\partial}{\partial n} \mathbf{q}_{0}\right] \\
& \left.-\frac{\Lambda}{r_{1}} \frac{\partial r_{1}}{\partial n}\left[\frac{\partial}{\partial t} \mathbf{q}_{0}\right]\right\} d S .
\end{aligned}
$$

As before, $r_{1}$ connects the point of observation with the point $Q$ in the integration process. Subtracting this result from the aperture formula, one obtains the alternate expression

$$
\begin{array}{r}
4 \pi \mathbf{q}(P, t)=4 \pi \mathbf{q}_{0}-\left\{\mathbf{K}_{c}-\stackrel{i}{-} \nabla_{P} \oint_{\Gamma} \frac{1}{r_{1}}\left[\int^{t} \mathbf{q}_{0} d t\right] \cdot d \boldsymbol{\Gamma}\right. \\
-\oint_{\Gamma} \frac{1}{r_{1}}[\mathbf{q} \mathbf{I} \times d \mathbf{\Gamma}\} .
\end{array}
$$

$\mathbf{K}_{c}$ is the same as the $\bar{K}$ in the aperture formula except that the integration is now taken over the surface $S_{c}$ of the "black" screen and the contour integration follows in the opposite direction, in agreement with the right-hand rule relative to the obstacle surface. But the above quantity in brackets is precisely the initial aperture formula for the complementary screen. Hence, it is evident that the formalism rigorously predicts a principle of complementary vector disturbance,

$$
\mathbf{q}_{0}=\mathbf{q}+\mathbf{q}_{c},
$$

$\mathbf{q}_{c}$ now denoting the complex vector disturbance of the complementary screen. The scalar analog of this principle is verified by Eqs. (4) and (6a). Likewise, the significance of $\mathbf{E}_{0}, \mathbf{H}_{0}$, in (7) and (11) of the vector case is now apparent. ' The field components for the "black" circular disk are thus obtained from those for the circular aperture by subtracting the latter from the unperturbed field components given in Eq. (8). This completes the solution.

These results are corroborated next for diffraction by the black disk in the optical region of the spectrum. The appropriate approximations are $\lambda \ll r_{0}, \boldsymbol{r}_{1}, a$. In (12) and (13) one introduces the simplifications $\Phi_{0}{ }^{\prime} \doteq-2 \pi j \Phi_{0} / \lambda$, $\Phi_{0}{ }^{\prime \prime}=-4 \pi^{2} \Phi_{0} / \lambda^{2}$, with similar expressions for $\Phi_{1}$, and omits terms like $\Phi_{0} \Phi_{0}{ }^{\prime} r_{0}{ }^{-1},\left(\Phi_{0}\right)^{2} r_{0}{ }^{-2}$, etc., compared with terms like $\Phi_{0}{ }^{\prime \prime},\left(\Phi_{0}{ }^{\prime}\right)^{2}, \Phi_{0}{ }^{\prime} \Phi_{1}{ }^{\prime}$, etc. To obtain an estimate on the error incurred in this procedure let us choose a local wavelength $\lambda=5 \times 10^{-5} \mathrm{~cm}$ (green light) and $r_{0}$, $r_{1} \gg 1 \mathrm{~cm}$. Then each term omitted contributes a fractional error not greater than one part in 20000 . Recalling that $\mathbf{E}(P)$ and $\mathbf{H}(P)$ are complex vectors, the time averaged Poynting vector in the $-x$ direction is $I_{-x}=-\hat{\imath}_{x} \cdot(c / 16 \pi)\left(\mathbf{E} \times \mathbf{H}^{*}+\mathbf{E}^{*} \times \mathbf{H}\right)$, with a similar

\footnotetext{
'The author wishes to correct a printing error which appears in the latter equation, reference 5 ; the second right-hand term in the expression for $4 \pi \mathbf{H}(P)$ should be multiplied by the gradient operator $\nabla_{p}$.
} 
expression $I_{-x 0}$ for the unperturbed field. Performing the above simplifications one thus obtains, for the $\sigma$-Case, the relative intensity at axial points behind the disk,

$$
\begin{gathered}
J_{-x}\left(x_{1}\right)=I_{-x} / I_{-x 0}=\left[\frac{a^{2}\left(x_{0}-x_{1}\right)}{4 r_{0} r_{1}}\right]^{2} \cdot F_{H} F_{E}+0(\lambda), \\
F_{H}=\frac{1}{r_{0}}-\frac{1}{r_{1}}+\frac{2 x_{0}\left(x_{0}-x_{1}\right)}{r_{0}\left(r_{0} r_{1}+x_{0} x_{1}+a^{2}\right)} \\
F_{E}=\frac{x_{0}}{r_{0}}\left(\frac{1}{r_{0}}-\frac{1}{r_{1}}\right)+\frac{\left(2-a^{2} / r_{0}^{2}\right)\left(x_{0}-x_{1}\right)}{\left(r_{0} r_{1}+x_{0} x_{1}+a^{2}\right)} .
\end{gathered}
$$

Analogous to (5), this quantity is independent of the wavelength so long as $0(\lambda)$ remains negligible. Letting $x_{0} \rightarrow \infty$ there obtains

$$
J_{-x}=2^{-4}\left(r_{1} / a\right)^{-2}\left[\frac{2 a}{r_{1}+x_{1}}-\frac{a}{r_{1}}\right]^{2}
$$

for collimated incident light. Hence, $J_{-x}(0)=\frac{1}{16}$, which value is $\frac{1}{4}$ that of the scalar prediction for the identical geometrical disposition. Further, as $x_{1} \rightarrow-\infty, J_{-x} \rightarrow$ $1-0$. The predictions of (18) are shown in Fig. 2 by the dashed curve; the diffracted relative intensity has everywhere smaller values than those predicted by the scalar theory. Likewise, when $x_{0}=a,(17)$ shows that $J_{-x}(0)=0.003791$, which value increases to the asymptotic value $J_{-x}(-\infty)=0.5308$. On the scalar theory the latter value was shown to be 0.7286 .

Likewise, from (12), (13), and (16), one computes the relative intensity for the $\sigma$-Case at points of the aperture axis,

$$
\begin{aligned}
J_{-x}\left(x_{1}\right)=1+\left[\frac{a^{2}\left(x_{0}-x_{1}\right)}{4 r_{0} r_{1}}\right]^{2} F_{H} F_{E} & \\
& -\frac{a^{2}\left(x_{0}-x_{1}\right)}{4 r_{0} r_{1}}\left(F_{H}+F_{E}\right) \cos \frac{2 \pi}{\lambda} \\
& \times\left(r_{0}-x_{0}+r_{1}-\left|x_{1}\right|\right) .
\end{aligned}
$$

$\lambda$ is again the local wavelength. The second term is the diffracted relative intensity of $\mathrm{Eq}$. (17). That quantitative agreement with the scalar theory is obtained may be verified by comparing (19) with (6b), term by term.

Since the axis of the aperture represents a critical point on the lateral intensity distribution curve, the region of interest is expected to be sufficiently broad to permit of experimental verification. However, in the microwave region recent experimental data exist, only for plane incident waves, at points of observation not greatly removed from the neighborhood of the circular aperture. ${ }^{10-14}$ Further experimental data may be found in the papers of $\mathrm{H}$. Severin, ${ }^{15}$ and $\mathrm{J}$. Meixner and $\mathrm{W}$. Andrejewski, ${ }^{16}$ which treat on the incident plane wavefront. Additional references may be found in Meixner's paper treating on the incident dipole field, ${ }^{17}$ in the review article of $\mathrm{C}$. J. Bouwkamp, ${ }^{18}$ and in the 1953 proceedings of the McGill symposium. ${ }^{19}$ At optical wavelengths there are only qualitative experimental (photographic) data for both the aperture and disk; the precision of measurement is not comparable with that obtainable by present day electronic-photometric techniques. Microwave measurements for the circular aperture at finite source distances (point sources) have never been discussed to our knowledge. Moreover, the diffraction of microwaves by the disk has never been undertaken.

${ }^{10}$ H. L. Robinson, J. Appl. Phys. 24, 35 (1953).

${ }^{11}$ R. E. Houston and R. H. Noble, J. Appl. Phys. 22, 1295 (1951).

${ }_{12}$ M. J. Ehrlich, S. Silver, and G. Held, J. Appl. Phys. 26, 336-45 (1955).

${ }^{13}$ S. J. Buchsbaum, A. R. Milne, D. C. Hogg, G. Bekefi, and G. A. Woonton, J. Appl. Phys. 26, 706-15 (1955).

${ }_{14}$ C. L. Andrews, Optics of the Electromagnetic Spectrum

(Prentice-Hall, Inc., Englewood Cliffs, New Jersey, 1960),

${ }^{15} \mathrm{H}$. Severin, Z. Naturforsch. 1, $487-95$ (1946).

${ }^{16} \mathrm{~J}$. Meixner and W. Andrejewski, Ann. Physik 7, 157 (1950); W. Andrejewski, Naturwiss. 38, 406 (1951); W. Andrejewski, "Die Beugung electromagnetischer Wellen an der leitenden Kreisscheibe und an der Kreisförmigen Öffnung im leitenden ebenen Schirm," doctorate dissertation, Rheinische Westfälischen Technischen Hochschule, Aachen, Germany (1952).

$17 \mathrm{~J}$. Meixner, Ann. Physik 12, 227 (1953).

${ }_{18}$ C. J. Bouwkamp, "Diffraction Theory," Reports on Progress in Physics (The Physical Society, London, 1954), Vol. 17, pp. $35-100$

${ }^{19}$ The McGill Symposium on Micronave Oplics, edited by B. S. Karasik and F. J. Zucker (Air Force Cambridge Research Center, April, 1959). 